\title{
Change History on Soil Temperature under Ridge in Semi-acrid Areas of Loess Plateau
}

\author{
Youhu Zhu ${ }^{1}$, Xianzhong $\mathrm{Wu}^{2}$, Zhitong $\mathrm{Wang}^{3}$ and Zhihua Gao ${ }^{3}$ \\ ${ }^{1}$ Ningxia Farm Survey Design Institute (CO., LTD), Yingchuan, Ningxia, 750001; \\ ${ }^{2}$ Department of physics and Hydraulic engineering of Gansu Normal University for Nationalities, \\ Gansu, hezuo 747000; \\ ${ }^{3}$ Alxa Left Banner Agriculture and Animal Husbandry Bureau of Inner Mongolia, Inner Mongolia, \\ Alxa Left Banner 750300
}

\begin{abstract}
Purpose: Studies on all-year-round soil temperature changes under ridge in semi-acrid areas of loess plateau. Method: three ways are given such as (CK), (TF) and (FF) on the basis of outside test data and observation temperature is $165 \mathrm{~cm}$. Result: soil temperature changes is obvious in the depth of $15 \mathrm{~cm}-15 \mathrm{~cm}$, retardation happens in the depth of $5 \mathrm{~cm} \sim-5 \mathrm{~cm}$ in different treatment and its time increases with the depth, and the best temperature difference TF is higher than $\mathrm{FF}\left(8.75^{\circ} \mathrm{C}\right), \mathrm{CK}\left(2.25^{\circ} \mathrm{C}\right)$, and below $0^{\circ} \mathrm{C}$ is caused by covered film in the depth of $-75 \mathrm{~cm}$, without such atmosphere by $\mathrm{CK}$ in the depth of- $100 \mathrm{~cm}$. Conclusion: it works as lessons for region-related scientific studies, practical guidance on regional agriculture production scientifically.
\end{abstract}

Keywords: Ridge film; All-yaer-round coverage; Temperature measure; Change history; Loess plateau

\section{黄土高原半干旱区垄覆膜下土壤温度时空变化}

\author{
朱有虎 $1 ，$ 吴贤忠 2 王治同，高志华
}

(1. 宁夏农垦勘测设计院 (有限公司) 宁夏 银川 750001; 2. 甘肃民族师范学院 物理与水电工程系, 甘肃 合 作 747000; 3.内蒙古阿拉善左旗农牧局，内蒙古 阿拉善左旗 750300）

摘要：目的：研究黄土高原半干旱区垄覆膜全年覆盖下土壤温度变化规律。方法：以野外试验数据为基础, 设置裸地 $(\mathrm{CK})$ 、透明薄膜覆盖 $(\mathrm{TF})$ 与覆膜毛毛(FF) 三种处理, 观测深度为 $165 \mathrm{~cm}$ 。结果：土壤温度在深度 $15 \mathrm{~cm} \sim 15 \mathrm{~cm}$ 土壤温度 变化剧烈, 不同处理下在 $5 \mathrm{~cm} \sim 5 \mathrm{~cm}$ 内出现滞后且滞后时间随深度而变长, 最值温差 TF处理较FF、CK处理高 $8.75^{\circ} \mathrm{C}$, 和 $2.25^{\circ} \mathrm{C}$ 。 覆膜使 $-75 \mathrm{~cm}$ 处无负温, CK处理在- $100 \mathrm{~cm}$ 处无负温。结论: 对区域相关科学研究提供借鉴, 为区域农业生产的科学实施具 有指导作用。

关键词：起垄覆膜；全年覆盖；温度测量；时空变化；黄土高原 引言

黄土丘陵区光热资源丰富，水分供应不足，土地利用不合理，农业整体生产水平低，抗 灾能力差。因此, 黄土高原旱作农业常将水保工程技术 (地膜覆盖等) 和水保耕作技术 (起 垄耕作等）相结合运用于生产实践 ${ }^{[1]}$ 。近十年来，以沟垄覆膜体系为核心的旱作栽培技术在 西北雨养农业区有了长足发展, 其区域已涵盖黄土丘陵沟壑区、旱塬区及残塬区等, 我国也 已成为世界上地膜覆盖栽培作物面积最大的国家 ${ }^{[2]}$ 。因此, 探讨地膜覆盖下土壤温度的变化 具有重要的现实意义。 
国内外对于地膜覆盖下土壤温度的研究取得一系列研究成果。目前, 研究成果在时间方 面多侧重于在作物生育期内, 在土壤深度方面集中于 $0 \sim 25 \mathrm{~cm}$, 研究目的主要关注地膜覆盖下 作物生长、产量及土壤水热变化状况 ${ }^{[3-8]}$ 。秦红灵等研究认为在垂直方向上, 土壤温度随土层

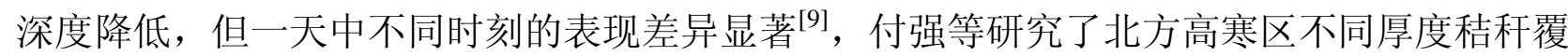
盖下 $0 \sim 140 \mathrm{~cm}$ 土壤温度的差异性 ${ }^{[10]}$ 。因此, 本文以陕西省米脂县起垄覆膜下土壤为研究对 象, 设置两类覆膜, 全年覆盖, 测定 $0 \sim 15 \mathrm{~cm}$ 的垄内温度与 $0 \sim 150 \mathrm{~cm}$ 的垄下温度, 分析不同 覆盖下土壤温度的时空变化，以期为黄土丘陵区生产实践提供理论与技术支撑。

\section{1 研究区概况及研究方法}

\section{1 研究区概况}

研究区位于中国典型黄土高原丘陵沟壑区陕西省米脂县远志山（东经 $108^{\circ} 49^{\prime} ，$ 北纬 $37^{\circ} 25^{\prime}$, 坡度为 $21^{\circ} \sim 39^{\circ}$ ) , 样地海拔 $890 \mathrm{~m}$, 属于中温带半干旱性气候区。根据米脂县多 年气象数据，该区年均气温 $8.5^{\circ} \mathrm{C}$, 积温 $3281^{\circ} \mathrm{C}$, 年辐射总量 $582.7 \mathrm{~kJ} / \mathrm{cm}^{2}$, 年日照时数 $2372.7 \mathrm{~h}$, 年均无霜期 $165 \mathrm{~d}$, 年均降雨量 $451.6 \mathrm{~mm}$, 主要集中在 7 9 三个月, 年潜在蒸发量 为 $1600 \mathrm{~mm}$ 左右, 属中温带半干旱气候。

试验区土壤为黄绵土，土壤容重 $1.29 \sim 1.31 \mathrm{~g} / \mathrm{cm}^{3}, 0 \sim 2 \mathrm{~m}$ 土层有效氮、磷、钾平均质量 比依次为 $30.12 、 1.56 、 89.33 \mathrm{mg} / \mathrm{kg}$, 有机质质量比 $2.1 \mathrm{~g} / \mathrm{kg}, \mathrm{PH}$ 值为 8.6 , 饱和持水量 $39.8 \%$, 田间持水量 $23.4 \%$ 。地下水埋深超过 $50 \mathrm{~m}$ 。

\section{2 研究方法}

\subsection{1 试验设计}

试验布设于研究区水平阶地上，布设时间为 2015 年 9 月，设计垄宽 $50 \mathrm{~cm} ，$ 垄高 $20 \mathrm{~cm}$ ， 垄上覆膜, 选取透明薄膜 ( TF ) 和覆膜毛毛 $(\mathrm{FF})$ 覆盖两种处理, $\mathrm{TF}$ 为常见的白色聚乙烯 农膜，以裸地无覆盖作为对照（CK），每个处理重复三次，9个小区随机区组排列。试验地 周边挖 $1 \mathrm{~m}$ 深槽, 埋设厚塑料布将周边土壤隔离, 以防止周边土壤水分及根系对试验小区产 生影响。

\subsection{2 数据采集}

土壤温度数据采集在两种覆膜地及裸地垄内 $15 \mathrm{~cm} 、 5 \mathrm{~cm} 、 0 \mathrm{~cm}$, 垄下 $5 \mathrm{~cm} 、 15 \mathrm{~cm} 、 30 \mathrm{~cm}$ 、 $50 \mathrm{~cm} 、 75 \mathrm{~cm} 、 100 \mathrm{~cm} 、 125 \mathrm{~cm} 、 150 \mathrm{~cm}$ （文中为区别垄内观测点, 将观测点深度表述为负 数) 处埋设 GS-3 探针, 利用 EM50 数据采集器进行采集记录, 步长为 $30 \mathrm{~min}$ 。测出土壤温 度指标单位为 ${ }^{\circ} \mathrm{C}$ 。距试验地 100 米处布设有小型自动气象站, 监测步长为 $10 \mathrm{~min}$, 用于测定 降雨 $(\mathrm{mm})$ 、气温 $\left({ }^{\circ} \mathrm{C}\right)$ 、净辐射 $\left(\mathrm{w} / \mathrm{m}^{2}\right)$ 、相对湿度 $(\%)$ 、风速 $(\mathrm{m} / \mathrm{s})$ 等。

\section{2 结果与讨论}

\section{1 黑白膜覆盖下的土壤温度日变化}

试验以2016年6月16日（晴天，其中14、15日晴，无降雨）观测数据为例。绘制24h内不 同覆膜处理下土壤温度在不同深度的垂直变化。从图1可看出, $15 \mathrm{~cm} \sim-150 \mathrm{~cm}$ 内, 土壤温度随 深度逐渐下降，FF、TF处理、CK对照日平均温度由 $28.02^{\circ} \mathrm{C} 、 32.7^{\circ} \mathrm{C} 、 25.73^{\circ} \mathrm{C}$ 降至 $17.2^{\circ} \mathrm{C}$ 、 
$16.23^{\circ} \mathrm{C} 、 15.69^{\circ} \mathrm{C}$ 。不同处理下同一深度内, $15 \mathrm{~cm}-15 \mathrm{~cm}$, 土壤温度变化剧烈, $-30 \mathrm{~cm} \sim-50 \mathrm{~cm}$ 变化平缓, $-75 \mathrm{~cm} \sim-150 \mathrm{~cm}$ 温度几乎不变。垄内土壤温度变化剧烈, 三种处理 $24 \mathrm{~h}$ 内温度最值都 出现在 $15 \mathrm{~cm}$ 处, 最值温度出现时间相同, 最小值 $14.45^{\circ} \mathrm{C} 、 15.9^{\circ} \mathrm{C} 、 9.95^{\circ} \mathrm{C}$ 出现在6:00, 最大 值 $43.05^{\circ} \mathrm{C} 、 53.25^{\circ} \mathrm{C} 、 45.05^{\circ} \mathrm{C}$ 出现在 $14: 00$, 三者最值温差为 $28.6^{\circ} \mathrm{C} 、 37.35^{\circ} \mathrm{C} 、 35.1{ }^{\circ} \mathrm{C}, 24 \mathrm{~h}$ 内 6: 00-14:00土壤温度呈上升趋势, 其余时间呈下降趋势。随土壤深度增加, 在 $5 \mathrm{~cm}$ - $5 \mathrm{~cm}$, 土壤温度最值时间相对于垄顶处都出现滞后现象, 最小值滞后1 2h, 而最大值滞后2 5小时, $-5 \mathrm{~cm}$ 深度处日平均土壤温度, $\mathrm{TF}$ 处理 $>\mathrm{FF}$ 处理 $>\mathrm{CK}$ 对照, 而夜间温度 $\mathrm{FF}$ 处理 $>\mathrm{TF}$ 处理 $>\mathrm{CK}$ 对照。 $-50 \mathrm{~cm}$ 深度处, 三者温度接近。

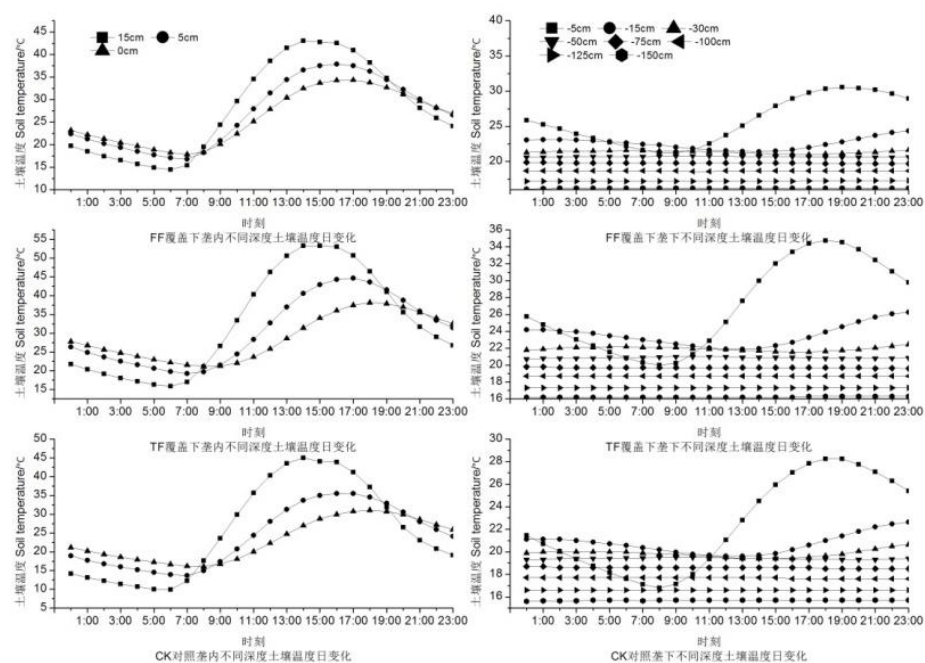

\section{2 不同处理下土壤温度剖面变化}

图 1 起垄覆膜下不同深度土壤温度日变化

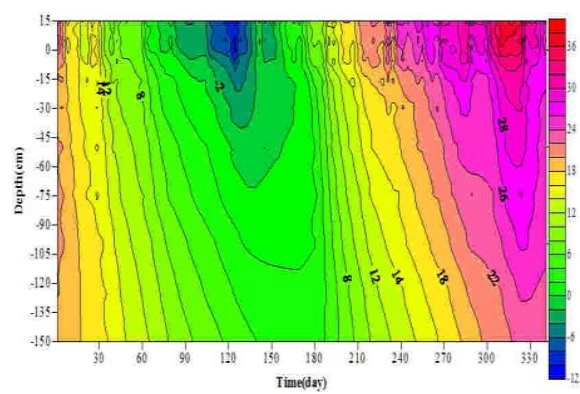

a.FF

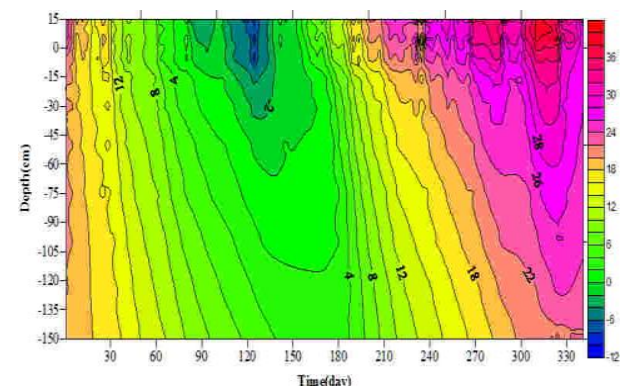

b.TF

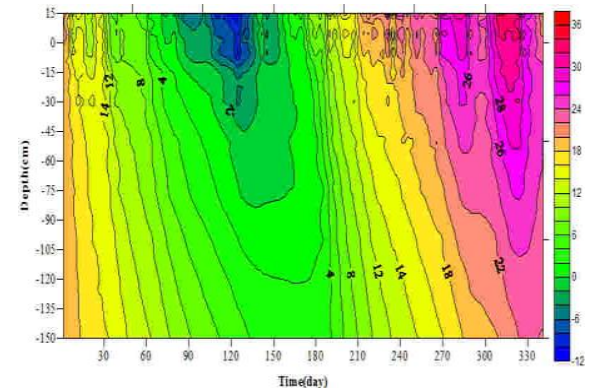

c. CK

图2 不同处理下土壤温度时空变化等值线图

试验观测时间为 2015 年 9 月 24 日-2016 年 8 月 29 日，累计 341d。观测时间内土壤温 度的时空变化特征可通过土壤温度等值线在空间上的疏密程度和随时间的平缓曲折程度来反 
映。不同覆膜处理下土壤温度时空变化等值线图见图 2. 地面覆盖改变了土气间的水热交换条 件 $^{[14]}$, 使得土壤温度变化滞后, 并在不同深度表现不一, 而土壤温度的改变引起土壤水分的 运移，造成部分土壤水分富集，进一步使得不同覆膜下土壤温度存在差异。如图 4 所示，在 15 - $15 \mathrm{~cm}$ 深度范围内等值线较密集, 表明土壤温度在该深度范围内变化剧烈, 在-30 - $150 \mathrm{~cm}$ 深度范围内, 土壤温度呈凹型分布。在 $-30 \mathrm{~cm}$ 与 $-50 \mathrm{~cm}$ 处, FF、 TF、 CK 处理下负温天数分布 为 $53 、 52 、 58$ 与 $32 、 27 、 42$, 覆膜使得 $-75 \mathrm{~cm}$ 处无负温, 而 $\mathrm{CK}$ 处理在该深度处负温达 37 天, CK 处理在 $-100 \mathrm{~cm}$ 处无负温。

\section{3 结论}

(1) 土壤温度随深度逐渐下降, 其变化为在深度 $15 \mathrm{~cm} \mathrm{-} 15 \mathrm{~cm}$ 土壤温度变化剧烈, $-30 \mathrm{~cm} \sim-50 \mathrm{~cm}$ 变化平缓, $-75 \mathrm{~cm} \sim-150 \mathrm{~cm}$ 温度几乎不变。不同处理下 $24 \mathrm{~h}$ 内 $6: 00-14: 00$ 土壤温 度呈上升趋势，其余时间呈下降趋势。三种处理下， $15 \mathrm{~cm}$ 处土壤温度最值出现时间相同，而 在 $5 \mathrm{~cm} \sim-5 \mathrm{~cm}$ 内出现滞后, 且滞后时间随深度而变长, 最值温差 $\mathrm{TF}$ 处理较 $\mathrm{FF}$ 处理高 $8.75^{\circ} \mathrm{C}$, 而 较 $\mathrm{CK}$ 高 $2.25^{\circ} \mathrm{C}$ 。

(2) 在15 -15cm深度范围内等值线较密集, 表明土壤温度在该深度范围内变化剧烈, 覆 膜使 $-75 \mathrm{~cm}$ 处无负温, $\mathrm{CK}$ 处理在 $-100 \mathrm{~cm}$ 处无负温。不同处理下负积温呈随深度减小的趋势, 三种处理下在 $0 \mathrm{~cm}$ 与 $-15 \mathrm{~cm}$ 深度处出现异常。

\section{4 致谢}

基金项目：甘肃民族师范学院院长科研基金项目：变化环境下甘南州水资源优化配置及生态 建设研究（13-10）；甘肃省高等学校科研项目：陇东黄土高原经济林主要树种耗水特性及 水分生产效益研究 (2015A-154)

\section{Acknowledgement}

Fund Project: Gansu Normal University for Nationalities project: Study on Optimal Allocation of Water Resources and Ecological Construction in Gannan under Changing Environment. (13-10); Gansu Higher Education Research Project: Study on Water Consumption Characteristics and Water Production Efficiency of Main Tree Species in Economic Forest of Longdong Loess Plateau. (2015A-154)

\section{参考文献:}

[1］周丽敏.黄土高原双垄覆膜和地槽集水技术对土壤水温、土壤养分及作物产量的影响 [D]. 兰州大学, 2009.

[2] 蒙强, 刘静霞, 张恒嘉. 我国黄土高原早作农业沟垄覆膜栽培技术研究进展 [J]. 贵州农业科学, 2015, 08:72-82.

[3] 江燕, 史春余, 王振振, 等. 地膜覆盖对耕层土壤温度水分和甘薯产量的影响 [J]. 中国生态农业学报, 2014, 06:627-634.

[4] 李荣, 王敏, 贾志宽, 等. 渭北旱塬区不同沟垄覆盖模式对春玉米土壤温度、水分及产量的影响 [J]. 农业工程学 报, 2012, 02:106-113.

[5] 李尚中, 王勇, 樊廷录, 等. 旱地玉米不同覆膜方式的水温及增产效应[J]. 中国农业科学, 2010, 05:922-931.

[6] Ramakrishna A, Tam H M, Wani S P, et al. Effect of mulch on soil temperature, moisture, weed infestation and yield of groundnut in northern Vietnam[J]. Field Crops Research, 2006, 95 (2/3): 115-125

[7] 李玉玲, 张鹏, 张艳, 等. 旱区集雨种植方式对土壤水分、温度的时空变化及春玉米产量的影响 [J]. 中国农业科 学, 2016, 06:1084-1096. 
[8] Zhou Limin, Li Fengmin, Jin Shengli, et al. How two ridges and the furrow mulched with plastic film affect soil water, soil temperature and yield of maize on the semi-arid Loess Plateau of China[J]. Field Crops Research, 2009, $113(1): 41-47$.

[9] 秦红灵, 高旺盛, 李春阳. 北方农牧交错带免耕对农田耕层土壤温度的影响 [J]. 农业工程学报, 2007, 01:40-47.

[10］付强, 马梓㮂, 李天霄, 等. 北方高寒区不同覆盖条件下土壤温度差异性分析 [J]. 农业机械学报, 2014, 12:152-159.

作者简介: 朱有虎 (1981一)，男，工程师，主要从事水资源高效利用研究。Emai1：wxz315@163.com。

\section{References:}

[1] Zhou Limin. Effect of Double RIDGES Covered with Plastic Film and Geosyncline Catchment Technology in Loess Plateau to Soil Temperature, Soil Nutrient and Crop Yield [D].Lanzhou University,2009.

[2] Meng Qiang, Liu Jingxia, Zhang Hengjia. Research Progress of Cultivation Technique for Ridge Overlay Film of Dry Farming in Loess Plateau [J].Guizhou Agricultural Sciences, 2015,08:72-82.

[3] Jiang Yan,Shi Chunyu, Wang Zhenzhen, etc.Effect of Plastic Mulch to Soil Temperature and Moisture of Plough Layer and Sweet Potato Yield [J].Chinese

[4] Journal of Eco-Agriculture,2014,06:627-634.

[5] Li Rong, Wang Min,Jia Zhikuan, etc. Effect of Different Furrow Cover Mode in Weibei Dry Land Area to Soil Temperature Moisture and Yield of spring corn [J].Transactions of Chinese Society of Agricultural Engineering,2012,02:106-113.

[6] Li Shangzhong, Wang Yong, Fan Tinglu, etc.Water Temperature and Fertilization Effect of Dry Land Corn in Different Overlay Film Ways [J].Scientia Agricultura Sinica,2010,05:922-931.

[7] Ramakrishna A, Tam H M, Wani S P, et al. Effect of mulch on soil temperature, moisture, weed infestation and yield of groundnut in northern Vietnam[J]. Field Crops Research, 2006, 95(2/3): $115-125$

[8] Li Yuling, Zhang Peng, Zhang Yan, etc. Effect of Rainwater Harvesting Planting Way in Drought Region to Temporal and Spatial Variation of Soil Moisture and Temperature and Yield of Spring Corn [J].Scientia Agricultura Sinica,2016,06:1084-1096.

[9] Zhou Limin, Li Fengmin, Jin Shengli, et al. How two ridges and the furrow mulched with plastic film affect soil water, soil temperature and yield of maize on the semi-arid Loess Plateau of China[J]. Field Crops Research, 2009, 113(1): 41-47.

[10]Qin Hongling, Gao Wangsheng, Li Chunyang. Effect of Zero Tillage in Interlock of Farming-Pastoral Region in Northern China to Soil Temperature [J].Transactions of Chinese Society of Agricultural Engineering,2007,01:40-47.

[11]Fu Qiang, Ma Zi'ao, Li Tianxiao, etc. Analysis on Soil Temperature Difference under Different Mulching Conditions in North Cold Area [J].Transactions of the Chinese Society of Agricultural Machinery,2014,12:152-159.

Author Brief: Zhu Youhu(1981-), male, engineer, chiefly work in highly utilization research on water resource. Email: wxz315@163.com. 